\section{Intervenções nutricionais e crescimento infantil em crianças de até dois anos de idade: uma revisão sistemática}

\author{
Nutritional interventions and child growth \\ among under-two-year-olds: a systematic review
}

\author{
1 Programa de Pós-graduação \\ em Epidemiologia, \\ Universidade Federal de \\ Pelotas, Pelotas, Brasil. \\ Correspondência \\ N. J. Valle \\ Programa de Pós-graduação \\ em Epidemiologia, \\ Universidade Federal de \\ Pelotas. C. P. 464, Pelotas, RS \\ 96001-970, Brasil. \\ njvalle@terra.com.br
}

\section{Abstract}

The aim of this study was to collect evidence of the impact of nutritional interventions on child growth. A systematic review of the literature on nutritional interventions in under-two-year-old children from 1980 to 2002 was conducted in the electronic databases (MEDLINE, LILACS, and MedCarib). The following descriptors were used: "nutrition", "child", "trial", "intervention", "growth", "infant", "programs", “impact", "counseling", "support", "body height", and "body weight". A complementary search was implemented by screening the bibliography cited in the previously located articles. Fourteen publications were found. The strategies used in the studies included distribution of nutritional supplements and/or nutritional counseling. Publication bias aside, most interventions presented a positive impact on child growth when applied during the first year of life. Nutritional counseling had the additional advantage of improving maternal and health professional practices on child nutrition and feeding.

Systematic Review; Infant Nutrition; Child Development
Neiva J. Valle 1

Iná S. dos Santos 1

Denise P. Gigante 1

\section{Introdução}

A desnutrição infantil é um importante problema de saúde pública em países em desenvolvimento, sendo considerada pela Organização Mundial da Saúde como uma das cinco principais causas de mortalidade infantil, além de ser subjacente às demais 1 . Estudos recentes têm mostrado que, entre grupos de crianças com distintas práticas alimentares, o crescimento infantil mantém-se similar a padrões de referência, até aproximadamente três a quatro meses de idade, começando então um progressivo declínio 2 . Este declínio coincide com o período usual de introdução de alimentos complementares 3 .

No Brasil, há evidências de que a amamentação não exclusiva nos primeiros seis meses de vida e após, no período dos 6 aos 24 meses, a monotonia da alimentação, acompanhada de alimentos de baixa densidade energética e pobres em micronutrientes, constitui-se em grave problema da alimentação infantil 4 .

A complexidade das questões envolvidas no crescimento infantil, seja quanto a quantidades adequadas de alimentos, questões comportamentais e até mesmo sócio-econômicas, específicas de cada localidade e diferente entre países, faz com que exista a necessidade de atualizar e adequar o conhecimento sobre $o$ assunto. A execução de intervenções é a técnica mais adequada para auxiliar a busca de respos- 
tas a esses questionamentos 5 . Nessa perspectiva, planejou-se realizar uma revisão sistemática de intervenções nutricionais, com o objetivo de instrumentalizar profissionais e gerentes do sistema de saúde para futuras intervenções nessa área. Foram rastreados artigos sobre estudos de delineamento experimental, do tipo intervenções nutricionais, dirigidas a crianças de zero a dois anos de idade, que avaliassem o crescimento infantil como desfecho principal. Uma revisão que aponte os principais elementos a serem prevenidos quando da condução de intervenções nutricionais poderá auxiliar no planejamento de futuros estudos e programas.

\section{Metodologia}

A estratégia de busca de artigos incluiu pesquisa em bases eletrônicas e busca manual de citações nas publicações inicialmente identificadas. Utilizaram-se as bases eletrônicas MEDLINE (National Library of Medicine, Estados Unidos), LILACS (Literatura Latino-americana e do Caribe em Ciências da Saúde) e MedCarib (produzida pela Rede Caribe), no período entre 1980 e 2002, período que concentrava a maior produção de intervenções nutricionais na infância. A revisão foi restrita a estudos com crianças menores de dois anos de idade, por ser esse o período do crescimento infantil de maior suscetibilidade a intervenções nutricionais.

As palavras "nutrition", “child”, "trial”, “intervention”, "growth”, “infant”, “programs”, “impact”, “counseling”, "support”, “body height” $\mathrm{e}$ "body weight" foram utilizadas como descritores. As referências bibliográficas dos estudos assim localizados foram também rastreadas para localizar outras intervenções de potencial interesse.

As intervenções selecionadas foram as que implementaram aconselhamento nutricional, complementação à amamentação e/ou suplementação alimentar. Excluíram-se os estudos que utilizaram somente medicamentos, suplementação com vitaminas, zinco, cálcio, ou fórmulas específicas, bem como aqueles realizados em grupos especiais de crianças, como de muito baixo peso ao nascer, pré-termo, hospitalizadas, com diarréia, anorexia nervosa ou outras morbidades.

Cada artigo foi classificado de acordo com os critérios de avaliação de Downs \& Black 6, que consideram cinco itens: informações disponíveis (objetivo, tipo de intervenção, desfe- cho, descrição da amostra, descrição das perdas, variabilidade aleatória dos resultados e probabilidade real dos achados), validade externa (representatividade da amostra, processo de amostragem, representatividade do local e do pessoal envolvido na intervenção), vieses (tipo de cegamento, planejamento prévio das análises, duração do acompanhamento em cada grupo, adequação da análise, adesão, acurácia dos testes), confusão (população de origem dos sujeitos, período de seleção, randomização, cegamento na alocação dos participantes, análises ajustadas, análise por intenção de tratar, perdas consideradas na discussão) e poder do estudo. O escore foi adaptado para intervenções comunitárias, excluindo-se os itens não aplicáveis, podendo totalizar 23 dos 31 pontos originais.

\section{Resultados}

Foram identificados 1.449 artigos com os descritores selecionados, localizando-se, a partir desses, 14 intervenções nutricionais dirigidas a crianças de zero a dois anos de idade, que avaliaram o crescimento infantil. A Tabela 1 apresenta um resumo dos estudos, os quais são apresentados em ordem cronológica de publicação. Seis dessas intervenções 7,8,9,10,11,12 foram conduzidas nas Américas; cinco em países asiáticos 13,14,15,16,17; uma no leste europeu 18; uma na África 19 e outra multicêntrica 20, envolvendo dois países africanos, um latino-americano e outro da Oceania. Sete intervenções 7,8, 10,11,13,15,20 utilizaram alimentos; quatro 12,14,16,18 foram baseadas em aconselhamento nutricional; duas forneciam um preparado especial ao grupo experimental 9,19 e apenas uma 17, alimento e aconselhamento nutricional, em dois grupos experimentais separados.

A idade das crianças, ao ingressarem nos estudos, variou de desde a gestação 7,9,14,16 até os 24 meses 8,15 ou sete anos 9. A maioria, no entanto, arrolou somente crianças menores de um ano de idade 7,10,11,16,17,18,19,20. Quanto ao estado nutricional, com exceção de dois estudos 11,18, em geral, foram arroladas crianças desnutridas 8 ou em risco nutricional 7,9,10,12,13, $14,15,16,17,19,20$. O período de acompanhamento das coortes variou de um mínimo de 11 semanas 10 a um máximo de sete anos 9 . A moda foi de acompanhar as crianças por seis a nove meses $11,12,15,17,19$ ou por pelo menos um ano $7,8,9,16,18$. 
Tabela 1

Intervenções nutricionais visando melhorar o crescimento infantil.

\begin{tabular}{|c|c|c|c|c|c|c|c|}
\hline Autor & Ano & País & Intervenção & $\begin{array}{l}\text { Grupo intervenção/ } \\
\text { grupo controle }\end{array}$ & $\begin{array}{l}\text { Tempo de } \\
\text { acompanhamento }\end{array}$ & $\begin{array}{l}\text { Efeitos } \\
\text { encontrados }\end{array}$ & Escore* \\
\hline Mora et al. 7 & 1981 & Colômbia & $\begin{array}{l}\text { Alimento para } \\
\text { gestantes, crianças } \\
\text { index e familiares }\end{array}$ & $\begin{array}{l}82 \text { com suplemento/ } \\
90 \text { sem suplemento }\end{array}$ & 3 anos & $\begin{array}{l}\text { > consumo calórico; } \\
\text { > peso da criança } \\
\text { de } 3 \text { a } 36 \text { meses; } \\
\text { > comprimento da } \\
\text { criança de } 6 \text { a } 36 \text { meses }\end{array}$ & 16 \\
\hline Walker et al. 8 & 1991 & Jamaica & $\begin{array}{l}\text { Alimento para criança } \\
\text { de } 9-24 \text { messes } \\
\text { e familiares }\end{array}$ & $\begin{array}{l}64 \text { com suplemento/ } \\
63 \text { sem suplemento }\end{array}$ & 12 meses & $\begin{array}{l}\text { > ganho de peso da } \\
\text { criança em } 6 \text { e } 12 \\
\text { meses; > ganho de } \\
\text { comprimento da criança } \\
\text { em } 6 \text { e } 12 \text { meses }\end{array}$ & 19 \\
\hline Husaini et al. 13 & 1991 & Indonésia & $\begin{array}{l}\text { Alimento em creches } \\
\text { para crianças de } \\
6-20 \text { meses }\end{array}$ & $\begin{array}{l}75 \text { de } 9 \text { creches com } \\
\text { suplemento/38 de } 11 \\
\text { creches sem suplemento }\end{array}$ & 14 semanas & $\begin{array}{l}\text { > consumo calórico; } \\
\text { > escore-Z peso/idade } \\
\text { em } 14 \text { semanas }\end{array}$ & 20 \\
\hline Brown et al. 14 & 1992 & Bangladesh & $\begin{array}{l}\text { Mensagens nutricionais } \\
\text { para mães de crianças } \\
\text { de } 4-14 \text { meses }\end{array}$ & $\begin{array}{l}62 \text { de } 3 \text { povoados com } \\
\text { treinamento/55 de } 5 \\
\text { povoados com hábitos } \\
\text { usuais }\end{array}$ & 5 meses & $\begin{array}{l}\text { > quantidade e variedade } \\
\text { de alimentos; > escore-Z } \\
\text { peso/idade em } 5 \text { meses }\end{array}$ & 17 \\
\hline Schroeder et al. 9 & 1995 & Guatemala & $\begin{array}{l}\text { dois preparados (Atole } \\
\text { e Fresco) para gestantes } \\
\text { crianças de } 0-7 \text { anos } \\
\text { e comunidade }\end{array}$ & $\begin{array}{l}2 \text { povoados com Atole } \\
\text { (alta energia e alta } \\
\text { proteína)/2 povoados } \\
\text { com Fresco (baixa } \\
\text { energia sem proteína) }\end{array}$ & 7 anos & $\begin{array}{l}\text { > consumo calórico, } \\
\text { > peso da criança de } \\
3-12 \text { meses; > peso da } \\
\text { criança de } 12-24 \text { meses; } \\
\text { > comprimento da } \\
\text { criança de } 3-12 \text { meses; } \\
\text { > comprimento da } \\
\text { criança de } 12-24 \text { meses }\end{array}$ & 15 \\
\hline $\begin{array}{l}\text { John \& } \\
\text { Gopaldas } 15\end{array}$ & 1993 & Índia & $\begin{array}{l}\text { Alimento para crianças } \\
\text { de } 6-24 \text { meses }\end{array}$ & $\begin{array}{l}21 \text { com mingau } \\
\text { semilíquido/ } 21 \text { com } \\
\text { mingau espesso }\end{array}$ & 180 dias & $\begin{array}{l}\text { > consumo calórico em } \\
\text { maiores de } 1 \text { ano; } \\
\text { > ganho de peso da } \\
\text { criança em } 180 \text { dias; } \\
\text { > ganho de peso da } \\
\text { criança em } 180 \text { dias por } \\
\text { faixa etária (6-12 meses, } \\
13-18 \text { meses e } 19-24 \\
\text { meses); > ganho de } \\
\text { comprimento da criança } \\
\text { em } 180 \text { dias; > ganho } \\
\text { de peso da criança em } \\
180 \text { dias nas faixas etárias } \\
\text { de 6-12 meses e } 13-18 \text { mese }\end{array}$ & 13 \\
\hline Cohen et al. 10,22 & $\begin{array}{l}1994 / \\
1995\end{array}$ & Honduras & $\begin{array}{l}\text { Alimentos caseiros } \\
\text { introduzidos em } \\
\text { crianças de } 4 \text { meses } \\
\text { (exclusivamente } \\
\text { amamentadas) da 16ạ } \\
\text { a 26a semana }\end{array}$ & $\begin{array}{l}47 \text { com alimentos e } \\
\text { amamentação livre, } \\
44 \text { com alimentos e } \\
\text { mantendo freqüência } \\
\text { da amamentação/50 } \\
\text { exclusivamente } \\
\text { amamentadas }\end{array}$ & $\begin{array}{l}11 \text { semanas }+ \\
6 \text { meses }\end{array}$ & $\begin{array}{l}\text { Sem efeito dos } 4-12 \\
\text { meses }\end{array}$ & 18 \\
\hline $\begin{array}{l}\text { Simondon } \\
\text { et al. } 20\end{array}$ & 1996 & $\begin{array}{l}\text { Congo, } \\
\text { Senegal, } \\
\text { Bolívia e } \\
\text { Nova } \\
\text { Caledônia } \\
\text { (França) }\end{array}$ & $\begin{array}{l}\text { Alimentos introduzidos } \\
\text { em crianças de } 4 \\
\text { meses amamentadas }\end{array}$ & $\begin{array}{l}\text { Congo: 74/74; Bolívia: } \\
\text { 78/82; Senegal: 66/68; } \\
\text { Nova Caledônia: } 63 / 53\end{array}$ & 3 meses & $\begin{array}{l}\text { > ganho de comprimento } \\
\text { da criança de } 4-5 \text { meses } \\
\text { e de } 4-7 \text { meses no Senegal } \\
\text { > ganho de comprimento } \\
\text { da criança de } 5-6 \text { meses na } \\
\text { Bolívia; < peso da criança } \\
\text { no Congo aos } 6 \text { e } 7 \text { meses }\end{array}$ & al; \\
\hline
\end{tabular}

(continua) 


\begin{tabular}{|c|c|c|c|c|c|c|c|}
\hline Autor & Ano & País & Intervenção & $\begin{array}{l}\text { Grupo intervenção/ } \\
\text { grupo controle }\end{array}$ & $\begin{array}{l}\text { Tempo de } \\
\text { acompanhamento }\end{array}$ & $\begin{array}{l}\text { Efeitos } \\
\text { encontrados }\end{array}$ & Escore* \\
\hline Mehta et al. 11 & 1998 & $\begin{array}{l}\text { Estados } \\
\text { Unidos da } \\
\text { América }\end{array}$ & $\begin{array}{l}\text { Alimento introduzido } \\
\text { aos } 3 \text { e aos } 6 \text { meses } \\
\text { Alimento comercial e } \\
\text { alimento escolhido }\end{array}$ & $\begin{array}{l}\text { Aos } 3 \text { meses (cedo): } 36 \\
\text { comercial/35 escolhido; } \\
\text { aos } 6 \text { meses (tardio): } 40 \\
\text { comercial/36 escolhido }\end{array}$ & 9 meses & $\begin{array}{l}\text { sem efeito entre cedo } x \\
\text { tardio aos } 12 \text { meses de } \\
\text { idade; sem efeito entre } \\
\text { escolhido x comercial } \\
\text { aos } 12 \text { meses de idade }\end{array}$ & 18 \\
\hline Lartey et al. 19 & 1999 & Gana & $\begin{array}{l}\text { Quatro preparados } \\
\text { (com Weanmix ou com } \\
\text { Koko) introduzido em } \\
\text { crianças de } 6 \text { meses }\end{array}$ & $\begin{array}{l}53 \text { Weanmix; } 51 \\
\text { Weanmix + vitaminas + } \\
\text { minerais; } 52 \text { Weanmix }+ \\
\text { peixe; } 52 \text { Koko + peixe/ } \\
79+385 \text { de dois estudos } \\
\text { transversais }\end{array}$ & 6 meses & $\begin{array}{l}\text { sem efeito entre os } \\
\text { quaro grupos intervenção; } \\
\text { > escore-Z peso/idade } \\
\text { aos } 8,11 \text { e } 12 \text { meses de } \\
\text { idade; > escore-Z } \\
\text { comprimento/idade aos } 8, \\
10,11 \text { e } 12 \text { meses de idade }\end{array}$ & 19 \\
\hline Guldan et al. 16 & 2000 & China & $\begin{array}{l}\text { Aconselhamento em } \\
\text { visitas domiciliares } \\
\text { mensais para mães } \\
\text { de menores de } 1 \text { ano } \\
\text { e grávidas }\end{array}$ & $\begin{array}{l}250 \text { de vilas com } \\
\text { educação nutricional/ } \\
245 \text { de vilas controle }\end{array}$ & 1 ano & $\begin{array}{l}\text { sem efeito com menos } \\
\text { de } 1 \text { ano acompanha- } \\
\text { mento; > escore-Z peso/ } \\
\text { idade aos } 12 \text { meses; > } \\
\text { escore-Z comprimento/ } \\
\text { idade aos } 12 \text { meses }\end{array}$ & 17 \\
\hline Bhandari et al. 17 & 2001 & Índia & $\begin{array}{l}\text { Alimento para crianças } \\
\text { de } 4 \text { meses e aconse- } \\
\text { lhamento nutricional } \\
\text { para mães de crianças } \\
\text { de } 4 \text { meses }\end{array}$ & $\begin{array}{l}104 \text { suplementação; } \\
104 \text { aconselhamento/ } \\
104 \text { visitação, } 106 \text { sem } \\
\text { intervenção }\end{array}$ & $\begin{array}{l}8 \text { meses (16-52 } \\
\text { semanas) }\end{array}$ & $\begin{array}{l}\text { sem efeito aconselha- } \\
\text { mento; > peso da } \\
\text { criança suplementado } \\
\text { x visitação }\end{array}$ & 20 \\
\hline Santos et al. 12 & 2001 & Brasil & $\begin{array}{l}\text { Aconselhamento } \\
\text { nutricional para mães } \\
\text { de menores de } 18 \\
\text { meses }\end{array}$ & $\begin{array}{l}218 \text { de } 14 \text { postos } \\
\text { treinamento/ } 206 \text { de } \\
14 \text { postos atendimento } \\
\text { usual }\end{array}$ & 180 dias & $\begin{array}{l}\text { > ganho peso da criança } \\
\text { em maiores de } 12 \text { meses; } \\
>\text { ganho escore-Z peso/ } \\
\text { idade em maiores de } 12 \\
\text { meses; > ganho escore-Z } \\
\text { peso/comprimento em } \\
\text { maiores de } 12 \text { meses }\end{array}$ & 21 \\
\hline Kramer et al. 18 & 2002 & Belarus & $\begin{array}{l}\text { Aconselhamento para } \\
\text { mães de recém- } \\
\text { nascidas }\end{array}$ & $\begin{array}{l}8.547 \text { em } 16 \text { locais } \\
\text { treinamento/7.895 em } \\
16 \text { locais atendimento } \\
\text { usual }\end{array}$ & 1 ano & $\begin{array}{l}\text { > peso da criança de } 1 \\
\text { a } 9 \text { meses; > comprimento } \\
\text { da criança de } 2 \text { a } 9 \text { meses; } \\
\text { sem efeito aos } 12 \text { meses }\end{array}$ & 21 \\
\hline
\end{tabular}

* Escore de qualidade de acordo com Downs \& Black 6.

Dos sete estudos com utilização de alimentos, cinco 10,11,13,15,20 foram dirigidos somente à criança índex e dois 7,8, direcionados também para as demais crianças e/ou familiares vivendo no mesmo domicílio.

Dentre os critérios de Downs \& Black 6, as principais limitações dos estudos foram: não descartar os principais fatores de confusão, não apresentar os valores-p reais para os principais desfechos, falta de cegamento para avaliação do desfecho, verificação confiável da adesão à intervenção, falta de descrição do procedimento de aleatorização e não apresentar o poder do estudo para detectar diferenças entre os grupos. Os parágrafos a seguir descrevem brevemente os estudos analisados.

O primeiro foi conduzido na Colômbia com gestantes cujos bebês tivessem risco nutricio- nal 7. O grupo intervenção foi constituído por 82 crianças suplementadas desde o sexto mês de gravidez até três anos de idade. O grupo controle foi formado por noventa crianças não suplementadas. A suplementação consistia em leite em pó, pão com farinha enriquecida e óleo vegetal, fornecida para todos os membros da família com mais de um ano de idade, distribuída semanalmente, em uma unidade de campo, para ser consumida em casa. Crianças menores de um ano, dos 3 aos 12 meses de idade, ou a partir do desmame, se anterior aos três meses, recebiam leite integral em pó, juntamente com uma mistura comercial vegetal de alto valor protéico. Comprimidos de vitamina A e ferro foram dados às mães suplementadas, durante a gravidez, e às crianças index. As crianças suplementadas apresentaram melhores mé- 
dias de peso, a partir dos três meses $(5,18 \mathrm{~kg} \mathrm{x}$ $4,98 \mathrm{~kg} ; \mathrm{p}<0,05)$, até os 36 meses de idade $(12,35 \mathrm{~kg}$ x 11,88kg; p < 0,05), alcançando uma diferença máxima de $0,52 \mathrm{~kg}$, aos 24 meses $(\mathrm{p}<$ $0,01)$. As diferenças entre médias de comprimento foram estatisticamente significativas, a favor do grupo intervenção, a partir de seis meses $(0,90 \mathrm{~cm} ; \mathrm{p}<0,01)$ até os 36 meses de idade $(2,20 \mathrm{~cm} ; \mathrm{p}<0,005)$. Aos 36 meses, $20,6 \%$ das crianças do grupo controle estavam moderadamente desnutridas, em contraste com $8,8 \%$ das do grupo suplementado. Houve casos de desnutrição grave, no grupo controle, entre 6 e 12 meses de idade, enquanto que, entre as crianças do grupo suplementado, nenhum caso foi registrado.

Na Jamaica 8 , crianças desnutridas de 9 a 24 meses de idade, com escore comprimento para idade $<-2$ (referência National Center of Health Statistics) 21 , foram recrutadas com o objetivo de verificar os efeitos da suplementação alimentar e do estímulo psicossocial, no domicílio, sobre o crescimento, o desenvolvimento e a ocorrência de morbidades. As crianças $(n=129)$ foram estratificadas por sexo e idade $(\leq 16 \mathrm{me}$ ses e $>16$ meses) e aleatoriamente designadas para um de quatro grupos: controle $(n=33)$; com suplementação alimentar $(n=32)$; com estímulo ( $\mathrm{n}=30)$ e, com ambos os tratamentos ( $n=32$ ), emparelhadas com outra sem déficit nutricional. O suplemento alimentar consistia em $1 \mathrm{~kg}$ de alimento à base de leite, contendo $525 \mathrm{kcal}$ e $14 \mathrm{~g}$ de proteína/100g, fornecido semanalmente, no domicílio. Além disso, $1 \mathrm{~kg}$ de leite em pó e alimento à base de cereal era fornecido para cada um dos demais membros da família. Não houve diferença significativa nos resultados observados entre o grupo controle desnutrido e o grupo que recebeu somente estímulo psicossocial. O grupo suplementado e o que recebeu ambas as intervenções, também apresentaram resultados semelhantes. Os autores reuniram esses dois pares de grupos e analisaram como grupo não-suplementado $(n=64)$ e grupo suplementado $(n=63)$. Na análise ajustada, nos primeiros seis meses, o grupo suplementado teve melhores incrementos de comprimento $(6,50 \mathrm{~cm} \times 5,60 \mathrm{~cm} ; \mathrm{p}<0,001)$ e peso $(1,56 \mathrm{~kg}$ x 1,25kg; p < 0,01) do que o não-suplementado. No período total do estudo (0-12 meses), entre as crianças suplementadas, foram observadas diferenças de $0,38 \mathrm{~kg}$ em peso, $0,94 \mathrm{~cm}$ em comprimento e $0,29 \mathrm{~cm}$ em circunferência da cabeça, superiores ao grupo não-suplementado. As crianças suplementadas permaneceram mais baixas do que as sem déficit. O efeito da suplementação apareceu nos primeiros seis meses da intervenção e foi mantido nos seis meses subseqüentes, mas não diferiu estatisticamente entre os grupos no segundo período.

$\mathrm{Na}$ Indonésia 13 , foi realizado um estudo com o objetivo de avaliar o efeito da suplementação alimentar sobre o desenvolvimento infantil, ao longo de 14 semanas. Foram estudadas 113 crianças com idades de seis a vinte meses, em 22 creches emparelhadas e aleatorizadas para o grupo intervenção, com suplementação além da dieta usual $(\mathrm{n}=75)$, ou controle, com dieta usual $(n=38)$. As porções incluíam arroz, farinha de arroz, farinha de trigo, pão, mandioca, batata, água de coco, açúcar e óleo, sendo fornecidas nas creches (duas vezes ao dia, seis dias por semana). A alimentação suplementar teve efeito significativo sobre a variação de escore- $Z$ de peso para idade, sendo de 0,29 no grupo suplementado e de -0,01 no grupo controle $(\mathrm{p}<0,001)$. Ambos os grupos possuíam, no arrolamento, marcado retardo de crescimento, sendo suas médias de escore- $Z$ de comprimento para idade de $-2,34$ e $-2,42$, respectivamente, no grupo suplementado e no não-suplementado. Levando em conta essa situação de risco nutricional, o efeito encontrado somente no ganho de peso, sugere que a duração da intervenção tenha sido demasiado curta para poder detectar efeitos no crescimento linear.

A intervenção em uma área rural de Bangladesh 14 incluiu 117 crianças com idades de 4 a 14 meses. O objetivo principal era melhorar a adequação da ingestão de nutrientes e, conseqüentemente, o estado nutricional e o crescimento de crianças amamentadas, através de mensagens nutricionais. O estudo teve duração de cinco meses e incluiu 62 crianças no grupo intervenção e 55 controles. Basicamente, consistia em demonstrações, no domicílio, de como enriquecer a alimentação através da adição de óleo, xarope denso de açúcar, leite, peixe, farinha de lentilha, vegetais e frutas da estação; além do estímulo à introdução de alimentos novos e à continuação da amamentação. Eram também discutidas mensagens para melhorar a higiene, como, por exemplo, o adequado armazenamento dos alimentos e a necessidade de lavar as mãos e recipientes, antes e depois do preparo e da refeição da criança. A percentagem de crianças com desnutrição grave, no arrolamento, foi de $16,0 \%$ e $9,0 \%$, respectivamente, nos grupos intervenção e controle. Ao final do estudo, essas percentagens foram de $21,0 \%$ e $35,0 \%$, sendo que o aumento no grupo controle foi estatisticamente significativo. As crianças do grupo intervenção obtiveram melhor variação em escore- $Z$ de peso para idade do que as controles (respectivamen- 
te, $-0,19$ e $-0,65 ; \mathrm{p}<0,001)$, estando aquelas cerca de $0,46 \mathrm{~kg}$ mais pesadas.

O estudo realizado por Schroeder et al. 9 na Guatemala, recrutou 1.208 crianças. Todos os membros de quatro povoados receberam um suplemento líquido, denso, em centros de suplementação alimentar, duas vezes ao dia. Em dois povoados, o suplemento era líquido e com alto teor calórico e protéico (Atole) e, em outros dois, continha baixas calorias (Fresco). O objetivo do estudo foi verificar o impacto, por categorias de idade, da suplementação alimentar, sobre o comprimento e o peso, durante os primeiros sete anos de vida. Foram incluídas crianças com idades variando de zero a sete anos. Na análise, foram incluídas apenas as crianças acima de três meses de idade. Os ganhos de peso e comprimento, no primeiro (312 meses) e segundo (12-24 meses) anos de vi$\mathrm{da}$, foram maiores no grupo Atole. No terceiro ano (24-36 meses), as crianças diferiram significativamente apenas em comprimento. Durante o primeiro ano de vida, entre as crianças do grupo Atole, comparativamente às do grupo suplementado com Fresco, cada 100kcal/dia de suplemento resultou em ganhos adicionais de, aproximadamente, $9 \mathrm{~mm}$ no comprimento $(\mathrm{p}<0,001)$ e de $0,35 \mathrm{~kg}$ em peso $(\mathrm{p}<0,001)$. No segundo ano, esse benefício decresceu para $5 \mathrm{~mm}(\mathrm{p}<0,001)$ e $0,25 \mathrm{~kg}(\mathrm{p}<0,001)$, respectivamente.

Estudo na Índia 15 recrutou 42 crianças moradoras em favelas, as quais receberam suplementação alimentar, por 180 dias. As crianças foram emparelhadas em 21 duplas, de acordo com a idade e o peso, sendo oito pares de 6-12 meses, seis de 13-18 meses e sete de 19-24 meses. As crianças do grupo experimental receberam no domicílio (uma vez ao dia), uma espécie de mingau engrossado com farinha de trigo, enriquecido com açúcar e óleo, com alta densidade energética e de consistência líquida ou semilíquida. O grupo controle recebeu alimento similar mas com consistência espessa, quase sólida. Considerando todas as crianças, houve maiores incrementos de peso no grupo intervenção do que no grupo controle (respectivamente, $2,10 \mathrm{~kg}$ e 1,00kg; $\mathrm{p}<0,001$ ), bem como nos comprimentos (respectivamente, $6,10 \mathrm{~cm} \mathrm{e}$ $4,50 \mathrm{~cm} ; \mathrm{p}<0,05)$. Considerando por faixa etária, foram significativamente melhores os aumentos de peso, em quilos, nas crianças do grupo experimental do que do controle: $1,90 \times 0,90$ ( $\mathrm{p}<0,01)$, de 6 a 12 meses; 2,00 x 0,90 ( $\mathrm{p}<0,05)$ de 13 a 18 meses; e, de 2,30 x 1,30 (p < 0,001) de 19 a 24 meses. Quanto aos aumentos de comprimento, somente não foram significativamente melhores entre as crianças de 19 a 24 meses de idade. A modificação de um alimento quase sólido, para uma consistência mais fina, possibilitou que as crianças consumissem mais, melhorando o crescimento.

Em Honduras, Cohen et al. 10 realizaram uma intervenção em mães de recém-nascidos, recrutadas nos hospitais, no pós-parto, que desejavam amamentar, pelo menos, por 26 semanas. O objetivo do estudo era avaliar diferenças de consumo e de crescimento em crianças amamentadas e crianças que receberam alimentação complementar, dos quatro até seis meses de idade. Na 16a semana de vida, as crianças foram alocadas em três grupos: controle com amamentação exclusiva até $26 \underline{\text { a }}$ semanas ( $\mathrm{n}=$ 50); com introdução de alimentos sólidos e amamentação conforme a livre demanda ( $\mathrm{n}=$ 47); e, com introdução de alimentos e mantendo a mesma freqüência de mamadas que no recrutamento $(n=44)$. Os ganhos médios de peso, comprimento e escore- $Z$ de peso para idade foram similares entre os três grupos. Nos seis meses seguintes de acompanhamento (6-12 meses de vida) não houve diferença entre os padrões de crescimento dos grupos 22 .

Um estudo multicêntrico foi realizado por Simondon et al. 20, em quatro países em desenvolvimento: Congo, Senegal, Bolívia e Nova Caledônia. O objetivo era verificar o impacto de uma suplementação nutricional de curto prazo (dos quatro aos sete meses de idade), na prevenção do déficit de crescimento. Os quatro países utilizaram o mesmo protocolo. Foram incluídas crianças com quatro meses de idade, ainda amamentadas, com escore- $Z$ de comprimento para idade $>-2,5$ e escore- $Z$ de peso para idade $>-2$. No Congo, foram aleatorizadas 148 crianças (74 para o grupo suplementado e 74 para o controle). Nos demais países, os grupos intervenção e controle, foram constituídos, respectivamente, por 66 e 68 crianças, no Senegal; 78 e 82, na Bolívia; e, 63 e 53, na Nova Caledônia. O suplemento (semilíquido) fornecido no domicílio (duas vezes ao dia, sete dias por semana), continha trigo pré-cozido, milho, farinha de soja, leite em pó, óleo vegetal, açúcar e era enriquecido com minerais e vitaminas. No Senegal, as crianças suplementadas cresceram mais rapidamente, em comprimento, do que as do grupo controle, dos quatro aos cinco meses, $(2,30 \mathrm{~cm} \times 1,72 \mathrm{~cm} ; \mathrm{p}<0,01)$; e, na Bolívia, dos cinco aos seis meses, $(1,80 \mathrm{~cm} \mathrm{x}$ $1,89 \mathrm{~cm} ; \mathrm{p}<0,01)$. Dos quatro aos sete meses, a variação de comprimento foi maior somente no Senegal $(5,03 \mathrm{~cm} \times 4,55 \mathrm{~cm} ; \mathrm{p}=0,02)$. A média de peso não diferiu entre os grupos do Senegal, Bolívia e Nova Caledônia, em períodos intermediários do estudo. No Congo, a média 
de peso foi significativamente menor no grupo suplementado aos seis $(6,92 \mathrm{~kg} \times 7,38 \mathrm{~kg} ; \mathrm{p}<0,05)$ e aos sete meses $(7,37 \mathrm{~kg} \mathrm{x} \mathrm{7,74kg;} \mathrm{p} \mathrm{<} \mathrm{0,05);} \mathrm{e} \mathrm{o}$ incremento de peso, dos cinco aos seis meses, também foi menor $(0,30 \mathrm{~kg} \times 0,47 \mathrm{~kg} ; \mathrm{p}<0,01)$. No período total de três meses de estudo, houve somente impacto positivo sobre o comprimento, verificado apenas no Senegal, onde o consumo de suplemento e os incrementos em peso e comprimentos foram os mais baixos do estudo.

Mehta et al. 11, nos Estados Unidos, investigaram se a introdução precoce de alimentos sólidos ou o tipo de alimento (preparado comercialmente ou escolhido pelos pais) produziria diferenças no crescimento ou na composição corporal. Foram recrutadas 165 crianças brancas, desmamadas, com menos de três meses de idade. Aos três meses, foram aleatoriamente alocadas em quatro grupos: com alimentos sólidos comerciais aos três meses $(n=36)$, com alimentos sólidos comerciais aos seis meses $(n=40)$, com alimentos escolhidos pelos pais aos três meses $(n=35)$ e com alimentos escolhidos pelos pais aos seis meses $(n=36)$. As crianças do grupo comercial foram primeiramente suplementadas com um único tipo de cereal após com múltiplos grãos de cereais e, por fim, com frutas e vegetais. As demais crianças receberam, primeiramente, cereais e após, outros alimentos da escolha dos pais ou dos pediatras. Os grupos com introdução precoce ou tardia de alimentos não diferiam, aos três meses, quanto ao peso $(6,18 \mathrm{~kg}$ x $6,27 \mathrm{~kg})$ ou ao comprimento $(61,00 \mathrm{~cm}$ x $61,30 \mathrm{~cm})$. Também não havia diferença entre os grupos com alimento comercial ou escolhido pelos pais, quanto a peso $(6,21 \mathrm{~kg} \times 6,25 \mathrm{~kg})$ e comprimentos iniciais $(61,00 \mathrm{~cm} \times 61,20 \mathrm{~cm})$. Não houve diferenças no crescimento, aos 12 meses, entre crianças dos grupos que introduziram alimentos precoce ou tardiamente (aos três ou aos seis meses), nem entre crianças do grupo de alimento comercial ou escolhido pelos pais, introduzidos em qualquer idade.

Em Gana, Lartey et al. 19 desenvolveram um produto alimentar (Weanimix) como alternativa entre alimentos complementares. O Weanimix consistia de milho, soja e amendoim torrados, misturados e moídos, para ser adicionado ao leite. O alimento foi distribuído semanalmente para ser consumido no domicílio três vezes ao dia. Aos seis meses de idade, 208 crianças foram alocadas, aleatoriamente, em um dos quatro grupos de suplementação alimentar: Weanimix $(\mathrm{n}=53)$, Weanimix com vitaminas e minerais $(n=51)$, Weanimix com farinha de peixe $(n=52)$ ou alimento local denomina- do Koko (espécie de mingau feito de milho fermentado) com farinha de peixe $(n=52)$. O grupo controle foi formado por crianças de 6 a 12 meses de idade, avaliadas antes do período de recrutamento $(\mathrm{n}=79$ ) ou após as crianças da intervenção terem completado 12 meses de idade $(n=385)$. Os escores- $Z$ médios de peso para idade e de comprimento para idade, dos quatro grupos suplementados, não diferiram entre si, em qualquer idade, entre 6 e 12 meses. Os ganhos de peso e comprimento também foram similares entre os quatro grupos. Quando comparadas com as controles, as crianças suplementadas, reunidas em um único grupo, tiveram médias de escore- $Z$ de peso para idade, aos 8, 11 e 12 meses de vida, significativamente maiores; e, de comprimento para idade, aos 8 , 10, 11 e 12 meses. Aos 12 meses, as médias de escores- $Z$ de peso para idade e de comprimento para idade, das crianças suplementadas, comparadas com as controles foram, respectivamente, $-1,19 \mathrm{x}-1,71(\mathrm{p}<0,001)$ e $-0,63 \mathrm{x}-1,27$ $(\mathrm{p}<0,001)$.

Na China, Guldan et al. 16 realizaram uma intervenção, baseada em aconselhamento nutricional. Em vilas do grupo intervenção, foram realizadas três sessões de treinamento para educadores nutricionais que, durante um ano, visitaram, mensalmente, mães de crianças menores de um ano de idade. Nos primeiros oito meses do estudo, todas as gestantes foram também visitadas mensalmente. Ao longo de um ano, foram estudadas 245 crianças no grupo controle e 250 no grupo intervenção, incluindo os recém-nascidos das gestantes acompanhadas. Aos 12 meses de acompanhamento, as crianças do grupo intervenção apresentaram melhores escore- $Z$ de peso para idade $(-1,17 \mathrm{x}$ $-1,93 ; \mathrm{p}=0,004)$ e de comprimento para idade $(-1,32 \times-1,96 ; p=0,02)$ do que as controles. A intervenção teve sucesso em diminuir taxas de anemia, promover o conhecimento materno sobre nutrição, melhorar as práticas alimentares, as taxas de amamentação e o crescimento infantil, aos 12 meses de idade.

Bhandari et al. 17, na Índia, conduziram um estudo para verificar o impacto de duas intervenções sobre o crescimento. Foram identificadas todas as grávidas e todas as crianças menores de quatro meses de idade. Um total de 418 crianças foi alocado, aleatoriamente, para um de quatro grupos: com suplementação alimentar ( $\mathrm{n}=104)$, com aconselhamento nutricional ( $n=104)$, controle com visitação domiciliar para verificar morbidades $(\mathrm{n}=104)$ e controle sem visitação $(n=106)$, denominado de grupo sem intervenção. O suplemento consistia em leite em pó, cereal de trigo, açúcar, vita- 
minas e minerais, sendo fornecido duas vezes por semana, no domicílio. O grupo com aconselhamento nutricional recebeu, mensalmente, sessões de 30-45 minutos de orientação, com nutricionistas treinadas. Ao longo do estudo, de 16 a 52 semanas, o grupo suplementado ganhou, em média, 0,25kg (IC95\%: 0,02-0,48) a mais de peso do que o grupo com visitação apenas $(p<0,05)$. Nos períodos parciais de acompanhamento, somente houve impacto entre 26 e 38 semanas, com diferença de $0,25 \mathrm{~kg}$ a favor do grupo suplementado quando comparado ao grupo com visitação apenas $(\mathrm{p}<0,05)$. Nos demais períodos intermediários (16-26 e 38-52 semanas), não houve diferença de ganho de peso entre esses dois grupos. Não houve efeito significativo de ganho de peso no grupo com aconselhamento nutricional, quando comparado com o grupo com visitação ou com o grupo sem intervenção. Nenhuma das intervenções trouxe benefício quanto a ganhos de comprimento.

Santos et al. 12, no Brasil, em um estudo visando verificar os efeitos do aconselhamento nutricional sobre o crescimento, selecionaram 424 crianças, menores de 18 meses de idade. Vinte e oito postos de saúde foram emparelhados e alocados em dois grupos: um com atendimento usual da rede (grupo controle) e outro com aconselhamento nutricional (grupo intervenção), de acordo com a estratégia desenvolvida pela Organização Mundial da Saúde/United Nations Children's Fund, "Atenção Integrada as Doenças Prevalentes na Infância" (AIDPI). Os médicos dos 14 postos intervenção foram treinados em aconselhamento nutricional, de acordo com guias alimentares do AIDPI, adaptadas para a realidade local. $\mathrm{O}$ aconselhamento nutricional teve impacto positivo no crescimento para as crianças que ingressaram no estudo com um ano ou mais de idade: em 180 dias, o ganho de peso foi maior no grupo intervenção do que no grupo controle $(1,48 \mathrm{~kg}$ x $1,14 \mathrm{~kg}$; $\mathrm{p}<0,05)$, bem como os ganhos nos escores-Z de peso para idade $(0,25 \mathrm{x}-0,06$; $\mathrm{p}<$ $0,05)$ e de peso para comprimento $(0,40 \times 0,12$; $\mathrm{p}<0,05)$.

O estudo mais recente foi conduzido em Belarus, por Kramer et al. 18, visando comparar a promoção do aleitamento materno, de acordo com a "Iniciativa Hospital Amigo da Criança”, com as práticas usuais de alimentação infantil. Foram incluídas 17.046 crianças com peso ao nascer acima de $2.500 \mathrm{~g}$, sendo 8.865 no grupo intervenção e 8.181 no grupo controle. Trinta e quatro hospitais e suas clínicas associadas foram emparelhados e alocados em dois grupos: um com atendimento usual (grupo con- trole) e outro com a intervenção. A proporção de crianças exclusivamente amamentadas foi sete vezes maior no grupo intervenção, aos três meses, do que no grupo controle $(43,3 \%$ x $6,4 \%$; $\mathrm{p}=0,001$ ). O peso foi $0,06 \mathrm{~kg}$ maior no grupo experimental ( $\mathrm{p}=0,001)$, com um mês, e aumentou até o terceiro mês $(+0,11 \mathrm{~kg} ; \mathrm{p}<0,001)$, declinando a seguir, mas ainda significativamente maior até nove meses $(+0,06 \mathrm{~kg} ; \mathrm{p}=0,02)$. Essa diferença desapareceu aos 12 meses. O comprimento teve padrão similar ao peso, sendo significativamente maior no grupo intervenção no segundo mês $(+0,32 \mathrm{~cm} ; \mathrm{p}=0,03)$, aumentando até o terceiro mês $(+0,50 \mathrm{~cm} ; \mathrm{p}=0,001)$ e declinando, a partir daí, até o nono mês $(+0,31 \mathrm{~cm}$; $\mathrm{p}=0,04)$. Na análise como se fosse estudo observacional (abdicando da intenção de tratar), o grupo com amamentação prolongada apresentou escore- $Z$ de peso para idade, até os três meses, acima dos valores apresentados pelos dois grupos do estudo experimental, caindo progressivamente a partir daí. Diferentemente, no grupo desmamado, esse escore caiu substancialmente no primeiro mês, recuperandose e igualando-se aos demais grupos, aos seis meses e, ultrapassando-os aos 12 meses.

\section{Conclusões}

Nessa revisão, a grande heterogeneidade quanto à faixa etária das crianças estudadas, o tipo de intervenção e o tempo de acompanhamento preveniram a realização de uma meta-análise que sumarizasse o efeito das intervenções sobre o crescimento infantil. A maioria dos estudos encontrados nesta revisão mostrou efeitos positivos das intervenções sobre o crescimento infantil. Dos 14, apenas dois não relataram efeitos no crescimento: o de Honduras 10,22 e o dos Estados Unidos 11. Entre os 12 com impacto positivo sobre o peso e/ou comprimento, encontravam-se cinco dos sete que utilizaram alimentos, os dois que empregaram um preparado especial e os quatro que deram aconselhamento nutricional. $O$ estudo que utilizou ambas as estratégias, alimentos e aconselhamento nutricional, também teve impacto positivo sobre o crescimento, mas somente nas crianças do grupo com alimentos. Deve-se considerar na análise desses resultados, no entanto, a possibilidade de viés de publicação, o qual se caracteriza pelo fato de estudos com resultados positivos terem maior probabilidade de serem aceitos pelas revistas científicas 23 .

Quanto à faixa etária suscetível às intervenções, essa revisão evidenciou que a maioria dos estudos que incluíram crianças menores de um 
ano de idade logrou detectar efeito positivo sobre o peso, ainda no primeiro ano de vida 7,9, $16,17,18,19$. Esse efeito permanecia detectável no segundo e terceiro anos de vida, se o período de acompanhamento fosse longo o suficiente 7,9. Cinco desses estudos 7,16,18,19,20 detectaram ganhos em comprimento, no primeiro ano de vida, entre as crianças do grupo intervenção. No estudo do Brasil 12, que incluiu crianças em risco de desnutrição, foi detectado efeito significativo da intervenção sobre o peso, somente no segundo ano de vida, não tendo sido encontrado impacto da promoção da amamentação, em crianças menores de seis meses. O estudo em Belarus 18, que incluiu crianças com médias antropométricas acima da referência NCHS 21 , mostrou efeitos positivos da promoção da amamentação, no peso e no comprimento, respectivamente, a partir do primeiro e segundo meses de acompanhamento, desaparecendo aos 12 meses. A diferença do efeito do apoio à amamentação, observada entre os dois estudos, provavelmente seja devida à diferença nas taxas de prevalência de amamentação nos grupos controles dos dois países, substancialmente menor em Belarus.

Quanto à duração das intervenções, alguns estudos evidenciaram que, mesmo as de curto prazo (menos de seis meses) e independentemente do tipo (suplementação alimentar ou aconselhamento), podem trazer benefícios ao

Resumo

O objetivo desse estudo foi reunir evidências sobre a eficácia de intervenções nutricionais sobre o crescimento infantil. Através de revisão sistemática da literatura, em bases eletrônicas (MEDLINE, LILACS e MedCarib), rastrearam-se estudos de intervenção nutricional dirigidos a crianças menores de dois anos, publicados entre 1980 e 2002. Os descritores usados foram "nutrition", “child", "trial”, "intervention", "growth", "infant", "programs", "impact”, “counseling", "support", "body height" $e$ "body weight". Busca adicional foi feita através das referências dos artigos localizados. Foram encontradas 14 intervenções que utilizaram suplemento elou aconselhamento nutricional. A maioria evidenciou impacto positivo no crescimento, quando aplicada no primeiro ano de vida. Afastado o viés de publicação, o aconselhamento teve a vantagem adicional de melhorar as práticas maternas e dos profissionais de saúde em nutrição e alimentação infantis.

Revisão Sistemática; Nutrição Infantil; Desenvolvimento Infantil crescimento. Caulfield et al. 24 realizaram uma revisão de intervenções nutricionais, restrita à faixa etária de 6 a 12 meses de idade, que incluiu os estudos da Colômbia 7, Jamaica 8, Indonésia 13, Bangladesh 14 e Guatemala 9. Nessa revisão, o aumento de 65 a 300kcal/dia no consumo alimentar introduzia diferenças no estado nutricional, a favor dos grupos intervenção, que variaram de $-0,25$ a 0,46 pontos, em escores- $Z$ de peso para idade, e de $-0,04$ a 0,35 , em escores- $Z$ de comprimento para idade.

Efeitos sobre outros desfechos, como melhoras no aporte nutricional e nos percentuais de desnutrição entre as crianças intervenção, quando comparadas com as controles, foram também relatados. Quando a intervenção utilizava aconselhamento nutricional, ocorreram melhoras em alguns outros aspectos: práticas maternas, dieta infantil, redução de anemia e desempenho dos profissionais de saúde.

Em resumo, a aplicação desses conhecimentos em programas governamentais ou não governamentais direcionados a crianças menores de dois anos, deveria enfatizar, antes dos seis meses, a promoção do aleitamento materno exclusivo e, a partir daí utilizar o aconselhamento nutricional continuado, com ou sem suplementação alimentar, como forma de melhorar, de maneira sustentável, as práticas maternas de alimentação infantil.

\section{Colaboradores}

N. J. Valle participou na busca dos artigos de interesse, sistematizou os resultados, redigiu as versões inicial e final do artigo. I. S. Santos e D. P. Gigante revisaram a versão inicial e contribuíram na revisão da versão final. 


\section{Referências}

1. Gove S. Integrated management of childhood illness by outpatient health workers: technical basis and overview. The WHO Working Group on Guidelines for Integrated Management of the Sick Child. Bull World Health Organ 1997; 75 Suppl 1:724.

2. Shrimpton R, Victora CG, de Onis M, Lima RC, Blossner M, Clugstn G. Worldwide timing of grow faltering: implications for nutritional interventions. Pediatrics 2001; 107:E75.

3. World Health Organization. Management of childhood illness: counsel the mother. Geneva: Division of Diarrhoeal and Acute Respiratory Disease Control, World Health Organization; 1995.

4. Giugliani ERJ, Victora CG. Normas alimentares para crianças brasileiras menores de dois anos. Brasília: Organização Pan-Americana da Saúde, Organização Mundial da Saúde; 1997.

5. Kirkwood BR. Essentials of medical statistics. Oxford: Blackwel Scientific Publications; 1998.

6. Downs SH, Black N. The feasibility of creating a checklist for the assessment of the methodological quality both of randomized and non-randomized studies of health care interventions. J Epidemiol Community Health 1998; 52:377-84.

7. Mora JO, Herrera MG, Suescun L, de Navarro L, Wagner M. The effects of nutritional supplementation on physical growth of children at risk of malnutrition. Am J Clin Nutr 1981; 34:1885-92.

8. Walker SP, Powell CA, Grantham-McGregor SM, Himes JH, Chang SM. Nutritional supplementation, psychosocial stimulation, and growth of stunted children: the Jamaican study. Am J Clin Nutr 1991; 54:642-8.

9. Schroeder DG, Martorell R, Rivera JA, Ruel MT, Hábicht JP. Age differences in the impact of nutritional supplementation on growth. J Nutr 1995; 125 Suppl:1051S-9S.

10. Cohen RJ, Brown KH, Canahuati J, Rivera LL, Dewey KG. Effects of age of introduction of complementary foods on infant breast milk intake, total energy intake, and growth: a randomised intervention study in Honduras. Lancet 1994; 344: 288-93.

11. Mehta KC, Specker BL, Bartholmey S, Giddens J, Ho ML. Trial on timing of introduction to solids and food type on infant growth. Pediatrics 1998; 102 (3 Pt 1):569-73.

12. Santos I, Victora CG, Martines J, Gonçalves H, Gigante DP, Valle NJ, et al. Nutrition counseling increases weight gain among Brazilian children. J Nutr 2001; 131:2866-73.

13. Husaini MA, Karyadi L, Husaini YK, Sandjaja, Karyadi D, Pollitt E. Developmental effects of short-term supplementary feeding in nutritionally-at-risk Indonesian infants. Am J Clin Nutr 1991; 54:799-804.
14. Brown LV, Zeitlin MF, Peterson KE, Chowdhury AM, Rogers BL, Weld LH, et al. Evaluation of the impact of weaning food messages on infant feeding practices and child growth in rural Bangladesh. Am J Clin Nutr 1992; 56:994-1003.

15. John C, Gopaldas T. Evaluation of the impact on growth of a controlled 6-month feeding trial on children (6-24 months) fed a complementary feed of a high energy-low bulk gruel versus a high energy-high bulk gruel in addition to their habitual home diet. J Trop Pediatr 1993; 39:16-22.

16. Guldan GS, Fan HC, Ma X, Ni ZZ, Xiang X, Tang MZ. Culturally appropriate nutrition education improves infant feeding and growth in rural Sichuan, China. J Nutr 2000; 130:1204-11.

17. Bhandari N, Bahl R, Nayyar B, Khokhar P, Rohde JE, Bhan MK. Food supplementation with encouragement to feed it to infants from 4 to 12 months of age has a small impact on weight gain. J Nutr 2001; 131:1946-51.

18. Kramer MS, Guo T, Platt RW, Shapiro S, Collet, JP, Chalmers B, et al. Breastfeeding and infant growth: biology or bias? Pediatrics 2002; 110 (2 Pt 1):343-7.

19. Lartey A, Manu A, Brown KH, Peerson JM, Dewey KG. A randomized, community-based trial of the effects of improved, centrally processed complementary foods on growth and micronutrient status of Ghanaian infants from 6 to 12 mo of age. Am J Clin Nutr 1999; 70:391-404.

20. Simondon KB, Gartner A, Berger J, Cornu A, Massamba JP, San Miguel JL, et al. Effect of early, short-term supplementation on weight and linear growth of 4-7-mo-old infants in developing countries: a four-country randomized trial. Am J Clin Nutr 1996; 64:537-45.

21. National Center for Health Statistics. Growth curves for children birth-18 years. Washington DC: U.S. Government Printting Office; 1977.

22. Cohen RJ, Brown KH, Canahuati J, Rivera LL, Dewey KG. Determinants of growth from birth to 12 months among breast-fed Honduran infants in relation to age of introduction of complementary foods. Pediatrics 1995; 96 (3 Pt 1):504-10.

23. Rothman KJ, Greenland S. Modern epidemiology. 2nd Ed. Philadelphia: Lippincott-Raven Publishers; 1998.

24. Caulfield LE, Huffman SL, Piwoz EG. Interventions to improve intake of complementary foods by infants 6 to 12 months of age in developing countries: impact on growth and on the prevalence of malnutrition and potential contribution to child survival. Food and Nutrition Bulletin 1999; 20:183-200.

Recebido em 09/Jul/2003

Versão final reapresentada em 27/Abr/2004 Aprovado em 14/Jun/2004 